MICROGLIA

\section{Non-apoptotic caspases are no less deadly}

Caspases are proteases that are best known for triggering apoptotic cell death. However, a study in Nature shows a novel, non-apoptotic role for caspases in the activation of microglia and subsequent neurotoxicity.

In their resting state, microglia monitor the brain environment, and on encountering damaged neurons or

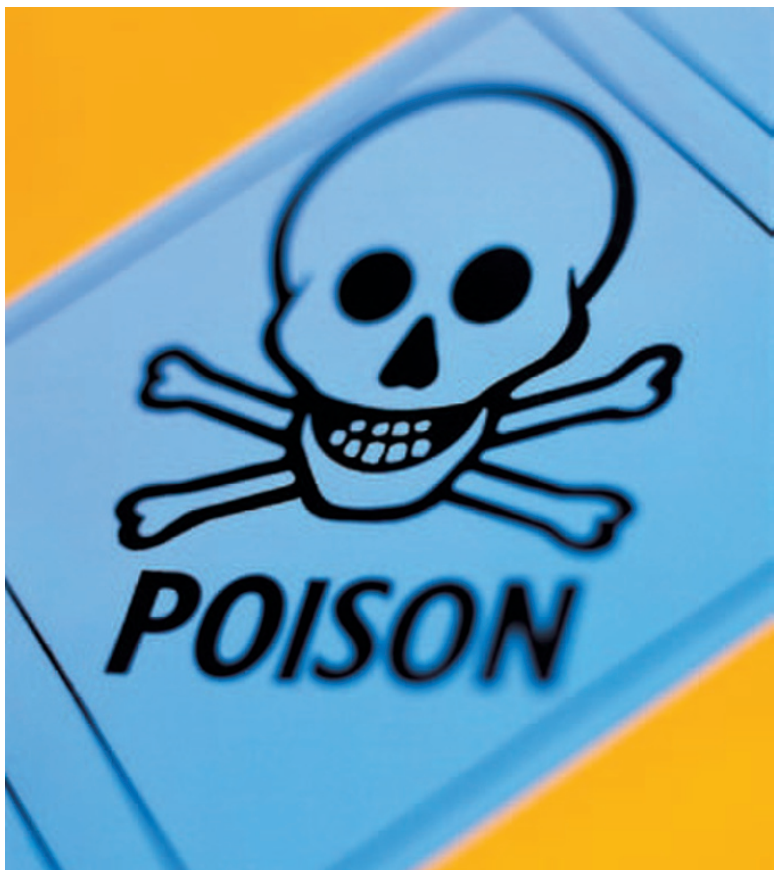

immunological stimuli they become active and release pro-inflammatory factors that can lead to further damage. Indeed, activated microglia are present in large numbers in CNS tissue from patients with neurodegenerative diseases including Alzheimer's disease, Parkinson's disease and amyotropic lateral sclerosis.

To investigate the signalling pathways that regulate activation of microglia, Joseph and colleagues exposed cultured microglia to lipopolysaccharide (LPS), a toll-like receptor 4 (TLR4) ligand that is found on Gram-negative bacterial walls. LPS treatment induced caspase 3 and caspase 7 cleavage and activation (identified by activation of the peptide DEVD) which, surprisingly, was not accompanied by microglial cell death. Activating microglia by exposing them to other proinflammogens, such as lipoteichoic acid, yielded similar results.

Treatment with an irreversible caspase inhibitor prevented the activation of microglia by LPS, and specifically silencing caspase 3 or caspase 7 with small interfering RNA (siRNA) also led to a decrease in DEVD activity and prevented activation of microglia. Further experiments to examine the events upstream and downstream of caspase 3 and caspase 7 cleavage showed that LPS treatment and TLR4 ligation activate caspase 8 , which in turn activates caspase 3 and caspase 7 , and that cleaved protein kinase $\mathrm{C}$ delta type $(\mathrm{PKC} \delta)$ is required for microglia activation downstream of caspase 3 and caspase 7 . Together, these findings point to a new, nonapoptotic role for caspase signalling in microglia.

To determine whether caspases mediate inflammation and neurotoxicity in the CNS, the authors co-cultured microglia with dopaminergic neurons. In these conditions, LPS treatment induces microgliamediated neuron death. However, knocking down caspase 3 or caspase 7 with siRNA reduced neurotoxicity. Moreover, caspase inhibition reduced microglia activation in rats injected with LPS and in a mouse model of Parkinson's disease. Finally, the authors detected high levels of active caspase 3 and caspase 8 in microglia from postmortem brain samples from individuals with Alzheimer's disease or Parkinson's disease. Together, these findings suggest that caspase inhibitors could hold promise in the treatment of neurodegenerative diseases.

Monica Hoyos Flight

ORIGINAL RESEARCH PAPER Burguillos, M. A. et al. Caspase signalling controls microglia activation and neurotoxicity. Nature 9 Mar 2011 (doi:10.1038/nature09788) 